\title{
Local Control of AC/DC Converters for Frequency Support Between Asynchronous AC Areas
}

\author{
Lampros Papangelis \\ Dept. of Elec. Eng. and Comp. Science \\ University of Liège, Belgium \\ 1.papangelis@ulg.ac.be
}

\author{
Marie-Sophie Debry \\ Patrick Panciatici \\ RTE, R \& D dept. \\ Versailles, France
}

\author{
Thierry Van Cutsem \\ Fund for Scientific Research (FNRS) at \\ University of Liège, Belgium \\ t.vancutsem@ulg.ac.be
}

\begin{abstract}
This paper proposes a novel control scheme for frequency support among asynchronous AC areas through HVDC grids. It is based on local controllers, each acting on a voltage source converter, using locally available measurements only, and supporting frequency of the adjacent $\mathrm{AC}$ area after a significant disturbance. The controller is combined with the existing DC voltage droop technique and is inspired of Model Predictive Control, taking into account various constraints. The coordination of the proposed control scheme with the existing secondary frequency control of an $\mathrm{AC}$ area is also discussed. Examples obtained from a test system with a five-terminal DC network connecting two asynchronous areas demonstrate the effectiveness of the proposed control scheme.

Index Terms-Multi-Terminal Direct Current grid, frequency support, secondary frequency control, voltage droop, Model Predictive Control.
\end{abstract}

\section{INTRODUCTION}

In contrast to AC interconnections, HVDC interconnected areas operate asynchronously and the primary reserves (or frequency containment reserves in [1]) of the one area do not participate in the frequency regulation of the other. Support between the two asynchronous AC areas can be enabled by providing the Voltage Source Converters (VSC) of the HVDC grid with dedicated controllers, which can be activated in emergency cases, in order to adjust the power transfer between areas through the HVDC grid [2].

Frequency support to an AC area by a Multi-Terminal Direct Current grid (MTDC) has been the subject of quite a number of publications. In the majority of them, a supplementary proportional (droop) control is added to the control structure of the VSC, enabling it to react to frequency deviations, e.g. in [3], [4]. A variant of the droop scheme was proposed in [5], where different droop values are used depending on the severity of the disturbance.

The drawback of the simple frequency droop control is the strong interaction with its DC voltage droop counterpart, which has been shown to reduce the efficiency of both control schemes [4].

In addition, frequency support through MTDC grids has been generally investigated under normal conditions assuming correct cooperation of the VSCs. It has not been tested in cases where some VSCs do not participate in DC voltage control as expected, which could result in significant DC voltage deviations. Furthermore, to the authors' knowledge, the study of frequency support to AC areas by MTDC grids has been limited to considering disturbances only inside the AC area. However, the loss of a VSC in an MTDC grid could also lead to significant frequency deviations, as well as severe DC voltage problems. In this case, it should be ensured that frequency control by the VSC can support frequency without aggravating the DC voltage profile of the system. Conventional control structures (e.g. a droop or a PI controller) would require a complex set of rules and correctly computed prespecified limits to take into account the above cases.

In this paper, the aforementioned problems are addressed by resorting to a novel control scheme for frequency support, inspired of Model Predictive Control (MPC) [6], [7]. MPC has received significant attention due to its ability to handle constraints, predict the system behavior and anticipate limit violations, which motivates its use in the present application. The proposed controller is tested for DC-side contingencies, which can lead to frequency problems in the neighboring AC areas, as well as for AC-side contingencies. In addition, the coordination of the proposed scheme with secondary frequency control of the AC areas (or frequency restoration process in [1]) is also considered.

The rest of the paper is organized as follows. Section II recalls the DC voltage droop control in MTDC grids. Section III details the formulation of the proposed control scheme. Section IV reports on simulations performed on a five-terminal MTDC grid. Concluding remarks are offered in Section V.

\section{DC VOLTAGE DROOP CONTROL}

This section briefly recalls the VSC DC voltage droop control, which interacts the most with the proposed control.

Controlling the DC voltages is of crucial concern for the correct operation of an MTDC grid. Indeed, in such a grid, power imbalances must be rapidly corrected, given the relatively small amount of energy stored in DC capacitors. Several methods have been proposed to this purpose. The DC voltage droop technique has received more attention [8] and has been adopted in this work. This method, inspired of AC frequency control practice, allows multiple converters to share any power imbalance affecting the MTDC grid while ensuring redundancy against the outage of one of them. In a droop-controlled MTDC grid some of the VSCs obey a $P-V$ characteristic defined by a power setpoint $P^{s e t}$, a voltage 
setpoint $V^{\text {set }}$ and a droop gain $K_{V}$. In steady state the VSC power $P$ is linked to the DC voltage $V$ through:

$$
P=P^{s e t}-K_{V}\left(V-V^{s e t}\right)
$$

where a positive power corresponds to rectifier operation. Therefore, following a power deficit in the MTDC grid, the DC voltage will start decreasing and the VSC will increase the power it injects into the DC grid until the balance is restored.

A simplified diagram of the VSC control structure based on the work in [9] is shown in Fig. 1, including the DC voltage droop control. The diagram focuses on the outer control loops which consist of the active and reactive power control. Active power varies according to the DC voltage of the VSC as described by Eq. (1). These control loops provide the active and reactive power commands ( $P^{c m d}$ and $Q^{c m d}$, respectively) to the current controller which then adjusts the modulation logic of the VSC. A Phase Lock Loop (PLL) is usually used to synchronize the VSC to the AC grid.

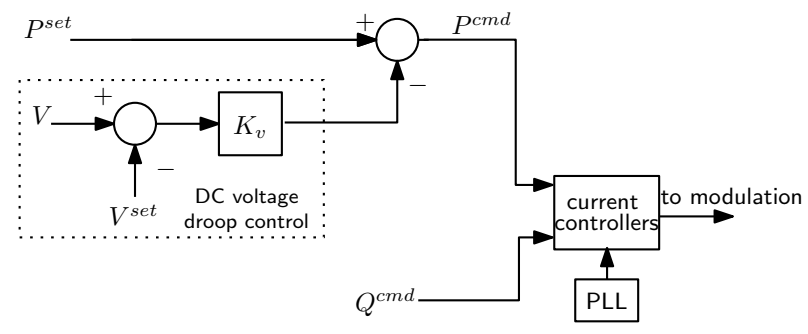

Fig. 1. Simplified diagram of the VSC control structure

\section{PROPOSED FREQUENCY CONTROL}

\section{A. Requested features of the control}

In this work, frequency support is considered as an "emergency" control scheme, as also suggested in [10]. Therefore, for small frequency deviations the frequency support scheme remains inactive, preventing continuous interactions between AC systems which were otherwise planned to operate asynchronously. In response to a large enough frequency deviation in one AC area, the VSCs connected to the latter sense the frequency deviation and correspondingly adjust the power transfer through the MTDC grid, thus taking advantage of the primary reserves of other AC areas.

The VSC is controlled to provide in steady state a predefined fraction of the total power injection needed to support frequency in the $\mathrm{AC}$ area of concern, as for a power plant under speed governor control. This can be achieved by changing the power setpoint $P^{s e t}$ of the $P-V$ characteristic (1) until the above objective is satisfied.

Clearly, the added control should not jeopardize the operation of the MTDC grid as well the other AC areas. This imposes to obey constraints on the DC voltage, on the rate of change of powers, etc.

Finally, it is highly desirable to rely only on local measurements readily available to each VSC. By so doing, fast and reliable performance can be achieved without resorting to

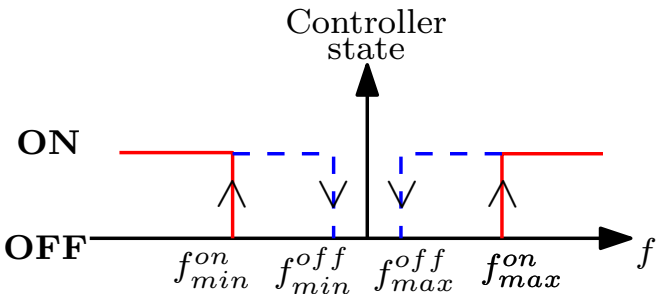

Fig. 2. Controller activation logic

extensive communication between converters, which can be subject to delays and failures.

\section{B. Constrained optimization problem}

For reasons mentioned in the Introduction, the proposed control relies on the MPC concept. This multi-step, optimization-based, closed-loop control scheme consists of computing a sequence of control changes which minimizes an objective and satisfies constraints in the future [6]. This optimization relies on a model of the future system evolution.

The MPC concept can be summarized as follows. At the current discrete time $k$, the controller has received the latest available measurements and has computed optimal control actions that have to be applied from $k$ up to the end of the control horizon $k+N_{c}-1$, so that the system meets a desired target at the end of the prediction horizon $k+N_{p}$. Out of this sequence, only the first component $\Delta \mathbf{u}(k)$ is applied. Then, at the next time instant $k+1$, the procedure is repeated for the updated control and prediction horizons, using the newly received measurements.

The proposed controller bears the spirit of an "emergency" scheme, thus being inactive in normal operation. Its activation is triggered by frequency deviations. As shown in Fig. 2, as long as frequency stays inside a pre-specified range $\left[f_{m i n}^{o n}, f_{m a x}^{o n}\right]$, the controller remains idle (OFF state), while it is activated as soon as frequency leaves the deadband (ON state). Once the controller has been activated, it remains active until the frequency is restored inside a narrower range $\left[f_{\min }^{o f f}, f_{\max }^{o f f}\right]$ by the secondary frequency controller of the AC system. This is further discussed in sub-section III-D.

Let $t^{\star}$ be the time when the control is activated, and $P$ the power injected by the VSC into the MTDC grid.

The main objective of frequency control is to adjust $P$ so that the steady-state participation of the VSC is proportional to the frequency deviation, i.e.

$$
\lim _{t \rightarrow \infty}\left[P(t)-P\left(t^{\star}\right)-K_{f}\left(f(t)-f_{N}\right)\right]=0
$$

where $f_{N}$ is the nominal frequency and $K_{f}$ the frequency droop gain.

The measurements used at time $k$ are:

$P^{m}(k)$ : the power flowing through the converter

$V^{m}(k)$ : the voltage at its DC bus

$f^{m}(k)$ : the frequency at its AC bus

$r_{f}^{m}(k)$ : the rate of change of frequency at its $\mathrm{AC}$ bus

They are either readily available in the converter sub-station or they can be obtained by the converter controllers (i.e. PLL). 
A reference evolution (or "trajectory" [7]) is defined with the objective of bringing the VSC power from its currently measured value to a value satisfying Eq. (2) in a finite number $N_{c}$ of control steps: for $j=1, \ldots, N_{c}$ :

$$
\begin{aligned}
& P^{r e f}(k+j)=P^{m}(k)+ \\
& \quad+\frac{j}{N_{c}}\left[P\left(t^{\star}\right)+K_{f}\left(f\left(k+N_{c}\right)-f_{N}\right)-P^{m}(k)\right] .
\end{aligned}
$$

It is easily checked by setting $j=N_{c}$, that the reference power at the end of the control horizon, $P\left(k+N_{c}\right)$, satisfies the participation defined by Eq. (2).

The constrained optimization at the heart of the proposed control consists in minimizing the deviations with respect to the above reference values:

$\min _{V, P, f, \epsilon, \Delta P^{s e t}} \sum_{j=1}^{N_{c}}\left[P^{r e f}(k+j)-P(k+j)\right]^{2}+v \sum_{j=1}^{N_{c}}[\epsilon(k+j)]^{2}$

subject to the following constraints: for $j=1, \ldots, N_{c}$ :

$$
\begin{gathered}
V^{\text {low }}(k+j)-\epsilon(k+j) \leq V(k+j) \\
V(k+j) \leq V^{u p}(k+j)+\epsilon(k+j) \\
\epsilon(k+j) \geq 0 \\
P^{\text {min }} \leq P(k+j) \leq P^{\text {max }} \\
V(k+j)=V(k+j-1)+s_{v} \Delta P^{\text {set }}(k+j-1) \\
P(k+j)=P(k+j-1)+\Delta P^{\text {set }}(k+j-1) \\
-K_{v}(V(k+j)-V(k+j-1))
\end{gathered}
$$

$f(k+j)=f(k+j-1)+\left[r_{f}(k)-s_{f}(P(k+j)-P(k))\right] T_{s}$

where $\Delta P^{s e t}$ is the change of VSC power setpoint, $\epsilon$ a slack variable, and $v$ a weight penalizing voltage violations. $T_{s}$ is the sampling time of the MPC.

Inequalities (5) and (6) specify that the DC voltage should not violate the limits $V^{\text {low }}$ and $V^{u p}$. In case the optimization problem becomes infeasible, these constraints are relaxed, with the $\epsilon$ variables taking nonzero values. However, $\epsilon$ is kept as small as possible by setting the weight $v$ to a high value. $V^{\text {low }}$ and $V^{u p}$ can evolve with time. so that they can smoothly restore the voltage inside the desired range, following a violation. This is further explained in sub-section III-C.

Constraint (8) imposes the VSC power to stay within limits.

Equations (9) and (10) make up the prediction model for the VSC powers and the DC voltages, initialized by setting the voltage (resp. power) to the last available measurement, i.e. $V(k)=V^{m}(k)$ (resp. $P(k)=P^{m}(k)$ ). The prediction horizon is taken equal to the control horizon $N_{c}$. The model is static, which is justified by the speed of action of power electronics and its controls, compared to the sampling period of the discrete controller (in the order of half a second). $s_{v}$ is the sensitivity of the DC voltage of a given VSC to the setpoint change $\Delta P^{\text {set }}$ of the same VSC. The value of $s_{v}$ depends on the voltage droop parameters of the VSCs and the topology of the MTDC grid.
Equation (11) gives the predicted $\mathrm{AC}$ system frequency values. These predictions assume that the measured rate of change of frequency $r_{f}^{m}(k)$ would remain constant, unless the DC power of the VSC is adjusted. Sensitivity $s_{f}$ relates the rate of change frequency at each time step $k+j$ to the VDC DC power change. The value of the sensitivity depends on an approximation of the inertia of the AC system, and the VSCs of the MTDC grid connected to it and participating in frequency support. Obviously, the aforementioned assumption neglects the actions of the prime movers of the synchronous machines, which would adjust their mechanical power production to contain the frequency drop. However, by choosing a short horizon for the MPC (i.e. a small value for the product $N_{c} T_{s}$ ), the prediction error can be relatively small. It should be emphasized, that all of the above approximations are easily compensated by the closed-loop nature of MPC.

The formulation can accommodate other constraints, such as maximum rate of change of power and/or DC voltage, maximum steady-state participation to frequency control, etc.

\section{Treatment of limit violations}

In normal operation, the DC voltage of the VSC lies between the minimum and maximum limits $V^{\min }$ and $V^{\max }$, respectively. In this case, the bounds in constraint (5) are: for $j=1, \ldots, N_{c}$ :

$$
V^{\text {low }}(k+j)=V^{\text {min }}, \quad V^{u p}(k+j)=V^{\max }
$$

However, it is possible that after a disturbance or due to the frequency support, the DC voltage of the VSC temporarily exceeds its normal operating limits. To avoid abrupt corrections the relevant bound is progressively tightened, starting from the measurement value, as follows: for $j=1, \ldots, N_{c}$ :

$$
\begin{aligned}
& V^{\text {low }}(k+j)=V^{m}(k)+\left(V^{\text {min }}-V^{m}(k)\right) \frac{j}{N_{c}} \\
& V^{u p}(k+j)=V^{m}(k)+\left(V^{\text {max }}-V^{m}(k)\right) \frac{j}{N_{c}} .
\end{aligned}
$$

It is easily checked by setting $j=N_{c}$ in Eqs. (13) and (14) that the value of the limits at the end of the control horizon are the specified secure values $V^{\min }$ and $V^{\max }$.

\section{Coordination with secondary frequency control}

Some works, e.g. [11], have investigated the possibility to use the MTDC grid in order to share the secondary reserves of the interconnected asynchronous AC areas. This is not the track followed in this work, where the existing secondary frequency control of each AC area [12] is left unchanged. Therefore, each $\mathrm{AC}$ area is solely responsible for restoring its frequency to its nominal value.

A simple model of secondary frequency control is used in this work as shown in Fig. 3. The controller calculates the Area Control Error (ACE) as follows:

$$
A C E=B \Delta f+\Delta P_{\text {tie }}
$$

where $B$ is the bias factor [12] and $\Delta P_{\text {tie }}$ the deviation of the total tie-line power from the scheduled value. For simplicity, 


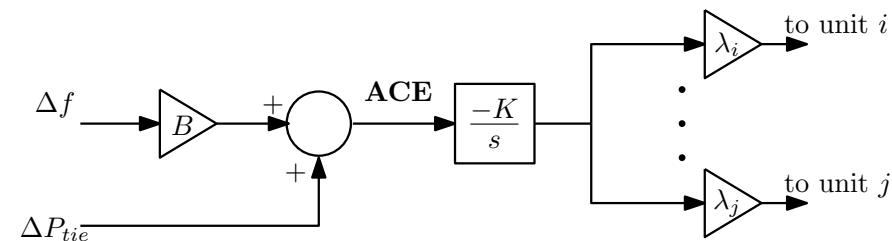

Fig. 3. Model of secondary frequency control

each AC system is assumed to make up a single control area and tie-line powers are not considered. The objective of secondary control is then to correct the frequency deviation.

The ACE is distributed to some generating units of the area according to participation factors $\lambda_{i}$.

Secondary control is deactivated when frequency enters a narrow deadband of width $2 \Delta f_{d b}$ around its nominal value, i.e. $\left[f_{N}-\Delta f_{d b}, f_{N}+\Delta f_{d b}\right]$.

The same deadband is used in this work to deactivate frequency support by the VSCs. Therefore, the values $f_{\text {min }}^{\text {off }}$ and $f_{\max }^{o f f}$ are chosen equal to $f_{N}-\Delta f_{d b}$ and $f_{N}+\Delta f_{d b}$, respectively. This choice serves a twofold purpose:

- the VSC controllers remain active and support the system frequency until normal operation is restored,

- from Equation (2), since $\lim _{t \rightarrow \infty} f=f_{N}$, the power of the frequency supporting VSC returns, if possible, to its pre-disturbance value $P\left(t^{\star}\right)$, thus also restoring the power exchange through the HVDC grid.

\section{Simulation REsults}

\section{A. Test system}

A simplified scheme of the test system is shown in Fig. 4. It consists of two asynchronous AC areas, one offshore wind farm and a five-terminal MTDC grid. Each AC area is based on the so-called Nordic test system, set up by an IEEE Task Force and detailed in [13], to which the reader is referred for a more detailed description. In both replicas, generator g20, which represented a large external AC system has been removed and the nearby equivalent load has been accordingly adjusted. Each AC area has two points of connection to the MTDC grid, in the North and the Central areas, respectively.

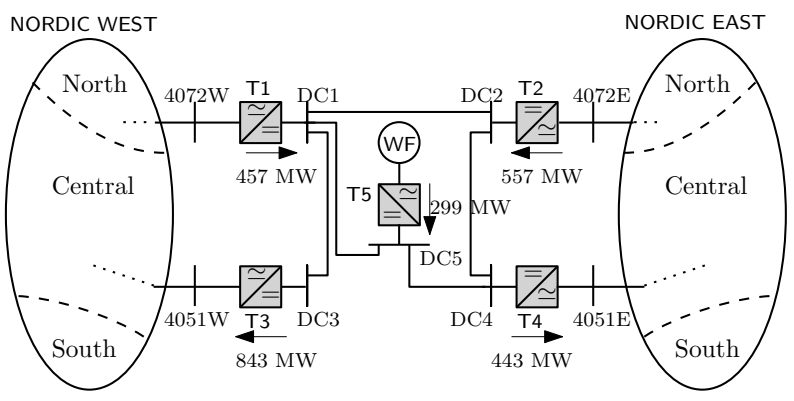

Fig. 4. Test system topology and initial power flow

All generators are represented with their automatic voltage regulators, excitation systems, speed governors and turbines as detailed in [13]. Each VSC is modeled in some detail with
28 differential-algebraic equations involving the phase reactor and DC capacitor dynamics, inner and outer control loops, PLLs, filters, etc. The DC branches are represented only by their series resistance by neglecting the series inductance and accounting for their DC capacitances in the terminal capacitors [14]. T5 is assumed to impose constant frequency and voltage on its AC side, thus acting as a slack bus for the offshore wind farm, merely modeled as a power injection.

Among the five VSCs, all but T5 operate in DC voltage droop mode, and are equipped with the proposed frequency control. A deadband of $\pm 200 \mathrm{mHz}$ is used for the activation of the controller. Frequency support stops when frequency reenters a range of $\pm 10 \mathrm{mHz}$ around its nominal value. T5 collects the power produced by the wind farm and injects it into the MTDC grid.

All discrete controllers have a sampling time $T_{s}=0.5 \mathrm{~s}$, which is long compared to the time constants of power electronics, but short with respect to frequency dynamics. In order to synchronize the VSCs acting on the same AC area, the controls $\Delta P^{s e t}$ are applied at discrete times $k T_{s}(k=$ $1,2, \ldots)$, assuming that each controller is relying on a GPSsynchronized clock. The measurements $P^{m}(k), V^{m}(k)$ and $f^{m}(k)$ are collected at times $k T_{s}-0.1 s(k=1,2, \ldots)$ to account for the time needed to solve the optimization problem.

The control and prediction horizons have been set both to $N_{c}=3$ to obtain a short enough time response. The weighting factor $v$ (see Eq. (4)) has been chosen equal to $10^{4}$.

The active power limits of each VSC have been set to $P^{\min }=-10$ and $P^{\max }=+10 \mathrm{pu}$ (on a $100 \mathrm{MW}$ base). The voltage limits at the DC buses of $\mathrm{T} 1-\mathrm{T} 4$ have been chosen equal to $V^{\min }=V^{o}-0.05$ and $V^{\max }=V^{o}+0.05$ $\mathrm{pu}$, where $V^{o}$ is the initial DC voltage.

Each area has its own dedicated secondary frequency control of the type described in Section III-D, whose objective is to restore the frequency in a range of $\pm 10 \mathrm{mHz}$ around its nominal value.

\section{B. Scenario 1: tripping of machine 88 in East subsystem}

The first scenario corresponds to the tripping of one generator in the East subsystem, which activates frequency control by $\mathrm{T} 2$ and $\mathrm{T} 4$. The system response to the event is shown in Figures 5-8.

Figures 5 and 6 show the frequencies in both $\mathrm{AC}$ areas, with and without frequency support from the VSCs. A closer look at the first $30 \mathrm{~s}$ reveals that frequency support by $\mathrm{T} 2$ and $\mathrm{T} 4$ yields a less pronounced frequency dip in the East system, as well as a mild drop of the frequency in the West system. In both cases, the frequencies of both $\mathrm{AC}$ areas are smoothly restored near their nominal values by secondary frequency control.

The power flows in converters T1-T4 are shown in Fig. 7. After activation of frequency control, $\mathrm{T} 2$ and $\mathrm{T} 4$ decrease their powers (i.e. inject more into the East $\mathrm{AC}$ grid) to support frequency. This is covered by $\mathrm{T} 1$ and $\mathrm{T} 3$, which increase their powers (i.e. draw more from the West AC grid) under the effect of voltage droop control. As already discussed, due 


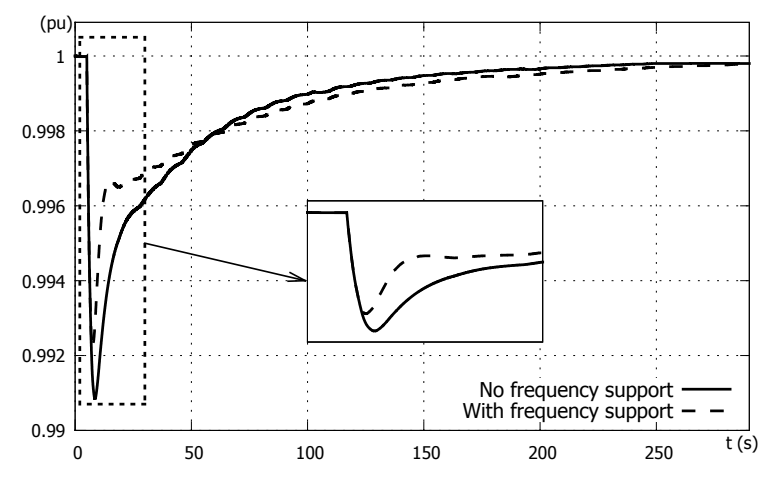

Fig. 5. Frequency of East area

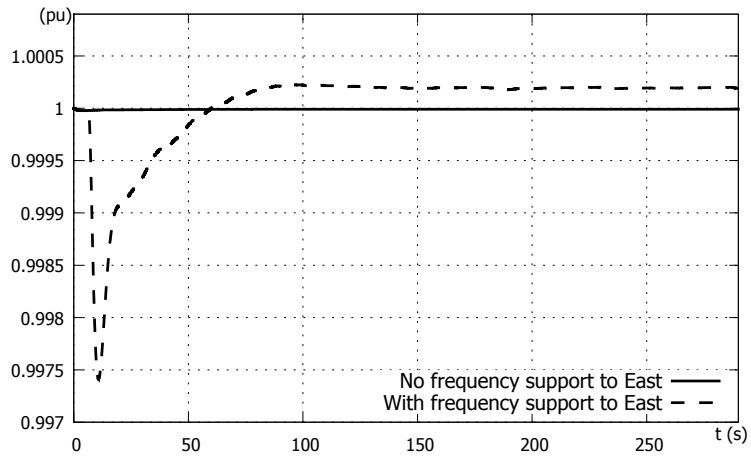

Fig. 6. Frequency of West area

to secondary frequency control, the powers of all VSCs are slowly restored at their pre-disturbance values.

The increased power demand by $\mathrm{T} 2$ and $\mathrm{T} 4$ will initially lead to a DC voltage drop, whose magnitude is dictated by the DC voltage droop gains $K_{v}$. The DC voltage response is shown in Fig. 8.

\section{Scenario 2: tripping of VSC T3}

The second scenario deals with a disturbance in the MTDC system, i.e. the tripping of terminal T3 at $t=5 \mathrm{~s}$. This event is followed by a very fast power adjustment of T1, T2 and T4, under the effect of DC voltage droop control. The outage is expected to cause a significant frequency deviation in both AC systems, but in opposite directions. Indeed, since the West system is missing the $843 \mathrm{MW}$ injected by T3, it will experience under-frequency. The East system, on the other hand, will experience over-frequency. In general, depending on the characteristics of each AC system (inertia, etc.), frequency support can be activated (i) only in the West subsystem, (ii) only in the East subsystem, (iii) in both subsystems.

Hereafter, the focus is on the first case, i.e. the behavior of the controller when frequency support is activated only in $\mathrm{T} 1$. Therefore, $\mathrm{T} 2$ and $\mathrm{T} 4$ are not equipped with the proposed controller for this scenario.

Figure 9 shows the frequencies of both systems with and without frequency support by $\mathrm{T} 1$. It can be seen that the frequency support activation slightly improves the response in both $\mathrm{AC}$ areas. As in the previous scenario, both frequencies

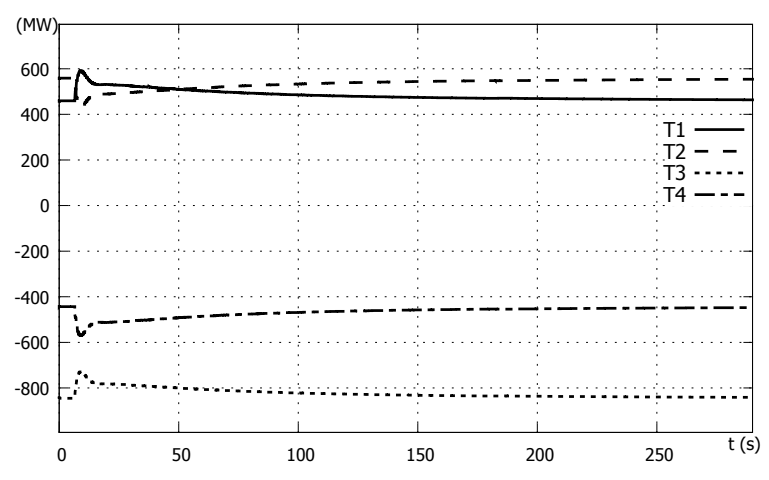

Fig. 7. VSC DC powers

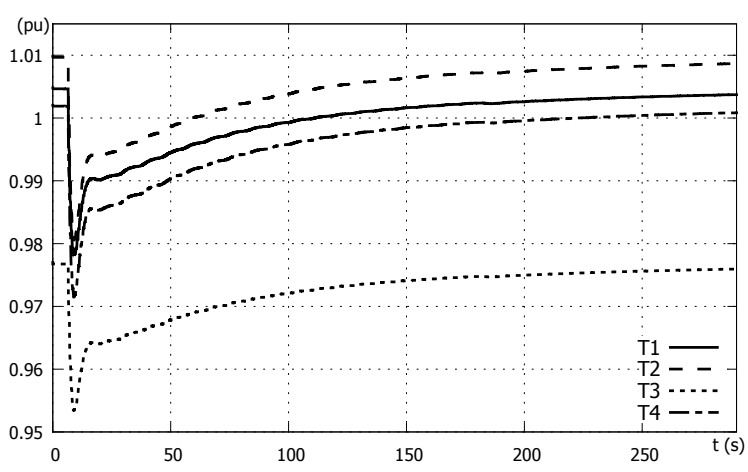

Fig. 8. MTDC grid DC voltages

are restored near their nominal values by the secondary frequency controller.

The DC powers of the VSCs are shown in Fig. 10. Due to DC voltage droop control, the powers of T1, T2 and T4 are quickly adjusted to restore the power balance in the MTDC grid. Then, at $t=7.5 \mathrm{~s}$, frequency support is activated in T1. At this point, the choice of the correct reference value $P\left(t^{\star}\right)$ has to be stressed. This value should be taken after the VSC power has settled under the effect of the DC voltage droop control. Otherwise, the VSC will not provide the desired participation that corresponds to the new configuration of the system. Given that the DC voltage response is much faster than the AC frequency response, it can be assumed that the MTDC grid will have reached a steady state before the frequency of the AC network exceeds the specified deadband. For this reason, it has been chosen to set $P\left(t^{\star}\right)$ to the last power measurement taken before the controller activation.

Finally, the DC voltages are shown in Fig. 11. Following the tripping of T3 they all rise very fast but are promptly stabilized by the DC voltage droop control. However, the DC voltage of T1 settles outside its limit. Therefore, following the activation of frequency support, the controller not only pursues to change the power of $\mathrm{T} 1$ to satisfy the desired participation, but also to bring the DC voltage below the maximum limit. Indeed, the voltage at bus DC1 eventually settles at its upper limit.

It fact, in this case the proposed controller automatically provides transiently more power than the one specified by the 


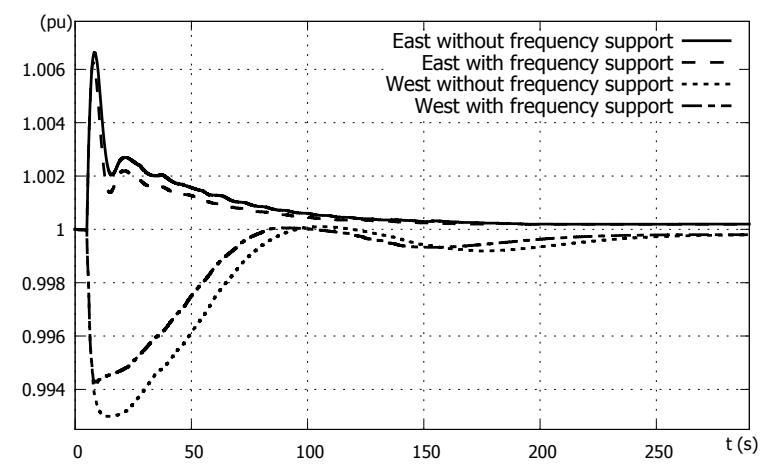

Fig. 9. Frequencies of East and West areas

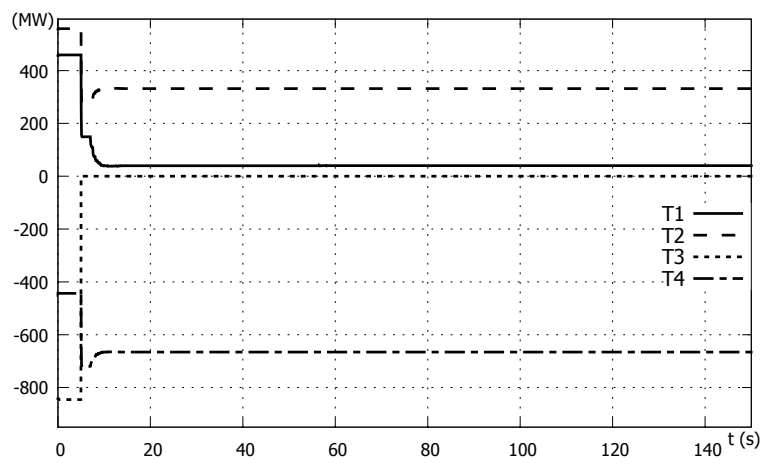

Fig. 10. VSC DC powers

desired droop gain. The reason is that the change of the T1 power setpoint required in order to bring the DC voltage to its limit is greater than the one required to satisfy the desired participation to frequency support. It is also important to note that in this scenario, the power of $\mathrm{T} 1$ does not return to its $P\left(t^{\star}\right)$ value following the restoration of the frequency by the secondary controller. It is prevented from doing so by the DC voltage constraint which has become active. This behavior is beneficial, since providing more power to the West system favors the response of the whole combined AC/DC system. Thus, it improves the frequency response of both East and West areas, it corrects the DC voltages, and T1 covers a larger part of the lost power.

\section{CONCLUSiON}

This paper has presented an MPC-based control scheme for primary frequency support among asynchronous $\mathrm{AC}$ areas. The proposed scheme aims at providing a pre-specified participation to the primary frequency regulation of an $\mathrm{AC}$ area, while explicitly taking into account the MTDC grid constraints, in particular the DC voltage limits. In addition, it relies purely on local measurements and no communication has been assumed between the AC/DC terminals.

Its cooperation with the existing conventional secondary frequency control has been also demonstrated, showing that by keeping the controller active while secondary frequency regulation is acting, the pre-disturbance power transfer between the two $\mathrm{AC}$ areas can be restored, unless a DC voltage

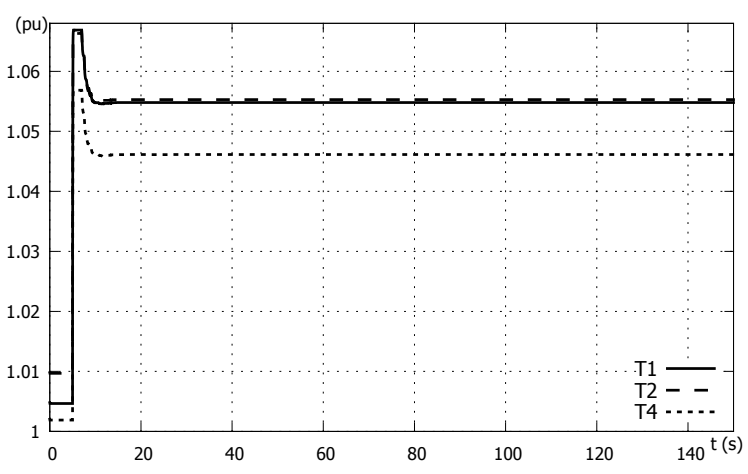

Fig. 11. MTDC grid DC voltages

constraint becomes active.

Future work includes further investigation of DC-side events, which could cause activation of frequency support in both systems at the same time, and the coordination with a centralized, slower MTDC grid control [15], aiming at monitoring the whole DC grid and coordinating the VSCs.

\section{REFERENCES}

[1] "ENTSO-e Network Code on Load Frequency Control and Reserves," 2013. Available online on https://www.entsoe.eu.

[2] D. Van Hertem and R. H. Renner, "Ancillary services in electric power systems with HVDC grids," IET Generation, Transmission \& Distribution, vol. 9, no. 11, pp. 1179-1185, 2015.

[3] N. R. Chaudhuri, R. Majumder, and B. Chaudhuri, "System Frequency Support Through Multi-Terminal DC (MTDC) Grids," IEEE Trans. on Power Systems, vol. 28, no. 1, pp. 347-356, 2013.

[4] S. Akkari, J. Dai, M. Petit, and X. Guillaud, "Coupling between the frequency droop and the voltage droop of an AC/DC converter in an MTDC system," in Proc. 2015 IEEE PES Eindhoven PowerTech, 2015.

[5] T. K. Vrana, L. Zeni, and O. B. Fosso, "Active power control with undead-band voltage \& frequency droop applied to a meshed DC grid test system," in Proc. 2012 IEEE ENERGYCON, 2012.

[6] J. M. Maciejowski, Predictive control: with constraints. Pearson education, 2002.

[7] S. J. Qin and T. A. Badgwell, "A survey of industrial model predictive control technology," Control Engineering Practice, vol. 11, no. 7, pp. 733-764, 2003.

[8] C. Dierckxsens, K. Srivastava, M. Reza, S. Cole, J. Beerten, and R. Belmans, "A distributed DC voltage control method for VSC MTDC systems," Electric Power Systems Research, vol. 82, pp. 54-58, jan 2012.

[9] P. Rault, Dynamic Modeling and Control of Multi-Terminal HVDC Grids. PhD thesis, Ecole Centrale de Lille, L2EP, 2014.

[10] L. Bizumic, R. Cherkaoui, and U. Häger, "Ch.17 - interface Protection," in Monitoring, Control and Protection of Interconnected Power Systems, pp. 333-347, Springer, 2014.

[11] D. Jing, Y. Phulpin, A. Sarlette, and D. Ernst, "Voltage control in an HVDC system to share primary frequency reserves between nonsynchronous areas," in Proc. 17th Power Systems Computation Conference 2011, 2011.

[12] P. Kundur, N. J. Balu, and M. G. Lauby, Power system stability and control, vol. 7. McGraw-hill New York, 1994.

[13] IEEE PES Task Force, "Test Systems for Voltage Stability Analysis and Security Assessment," 2015. Technical Report PES-TR19, Available online on IEEE PES Resource Center. http://resourcecenter.ieeepes.org/pes/product/technical-publications/PESTR19.

[14] L. Papangelis, X. Guillaud, and T. Van Cutsem, "Frequency support among asynchronous AC systems through VSCs emulating power plants," in Proc. 2015 11th IET International Conference on AC and DC Power Transmission, 2015.

[15] L. Papangelis, M.-S. Debry, P. Panciatici, and T. Van Cutsem, "Coordinated Supervisory Control of Multi-Terminal HVDC Grids: a Model Predictive Control Approach," IEEE Trans. on Power Systems, Early access, 2017. 